IWSCFF-Paper-2010-6-1

\title{
RELATIVE STATE ESTIMATION AND OBSERVABILITY FOR FORMATION FLYING SATELLITES IN THE PRESENCE OF SENSOR NOISE
}

\author{
D.C. Maessen ${ }^{1}$, E. Gill ${ }^{1}$ \\ ${ }^{1}$ Department of Space Engineering, Delft University of Technology, Kluyverweg 1, 2629 HS, \\ Delft, The Netherlands \\ d.c.maessen@tudelft.nl, e.k.a.gill@tudelft.nl
}

\begin{abstract}
This paper presents an investigation into the relative state estimation and observability for two formation flying satellites using two different relative navigation sensor sets. The first set consists out of a transmitter antenna on one satellite and a single receiver antenna on the other satellite to measure the inter-satellite range using a radiofrequency ranging signal. The second set uses three receiver antennas to measure multiple ranges, effectively providing angular information. It is derived in the paper that for the more complete sensor set, the error in the estimation of the relative state is a function of the pseudorange error, the inter-satellite distance, and the receiver antenna baselines. By varying these variables, conditions are found for which the observations obtained using the first sensor set result in relative state estimation and observability comparable to those obtained with the more complete sensor set. This offers potential reductions in cost and complexity for certain mission scenarios.
\end{abstract}

KEYWORDS : formation flying, inter-satellite ranging, noise, estimation, observability

\section{INTRODUCTION}

For the autonomous control of a satellite formation, the relative position and velocity of the satellites needs to be estimated. This can be done using ground-in-the-loop if the formation is relatively loose (i.e. large inter-satellite distances and large control windows). Alternatively, for tight formations, the required information needs to be obtained and processed onboard the satellites. In this work, we study the latter case. Two satellites, a chief and a deputy, perform inter-satellite range measurements using locally generated radiofrequency ranging signals to obtain the information required to estimate the relative position and velocity. The deputy, which is treated as a point mass, transmits a ranging signal to the chief, which uses either one or three receiver antennas to pick up the signal and determine the relative range(s). When the chief is equipped with three antennas, being separated by a known baseline, angular information is effectively present in the measurements which results in a more accurate navigation solution than when using a single antenna. However, since having fewer sensors on a satellite generally implies less complexity and thus less costs, it is of interest to investigate how many sensors are sufficient to meet certain mission requirements. Especially when faced with limitations in ranging accuracy and antenna baseline, the angular information obtained by using multiple antennas might be of such poor quality that the navigation solution is hardly better than what can be achieved using a single antenna.

To investigate this problem, we seek to determine to what extent the relative state of the two satellites, flying in formation in a low Earth orbit (LEO), can be estimated using a linearized relative dynamics model and noisy inter-satellite range measurements using the two different sensor sets. To isolate the properties we are interested in, we have implemented a very simple setting. More advanced and realistic effects are considered to provide only minor 
contributions and effects to our findings. The chief is orbiting in a perfectly circular orbit and it is assumed that the only force acting on the satellites is the gravity caused by a spherical Earth. The location of the receiving antennas with respect to the center of mass of the chief is assumed to be known perfectly and signal blockage by the satellite body is not considered. The range measurements are treated together with a dynamic model of the satellites' relative motion to estimate their relative orbit using an iterative batch least-squares (LSQ) algorithm.

The paper is organized as follows. Section 2 introduces the reference frames and orbital dynamics used to model the current problem. Section 3 describes the iterative batch LSQ algorithm that is used for the relative state estimation. In section 4 it is shown how the antenna baseline, ranging error, and inter-satellite range affect the relative position estimation. The results for various test cases are shown and discussed in section 5 while section 6 concludes this research.

\section{REFERENCE FRAMES AND RELATIVE ORBITAL DYNAMICS MODELING}

Since the relative orbital elements of two co-orbiting satellites are to be determined, an appropriate coordinate frame in which the relative motion can be described is the Hill frame [1]. This frame is centered at the chief with the radial unit vector $\boldsymbol{o}_{\mathrm{x}}$ aligned with the orbit radius vector, the normal unit vector $\boldsymbol{o}_{\mathrm{z}}$ aligned with the orbit angular momentum vector, and the tangential unit vector $\boldsymbol{o}_{\mathrm{y}}$ directed such that a right-handed Cartesian reference frame is formed, cf. Figure 1 . The body reference frame of the chief, with unit vectors $\boldsymbol{b}_{\mathrm{x}}, \boldsymbol{b}_{\mathrm{y}}$, and $\boldsymbol{b}_{\mathrm{z}}$, is centered at and aligned with the Hill frame, which is typical for, e.g., an Earth observation mission.

In the Hill frame, the relative motion of the deputy with

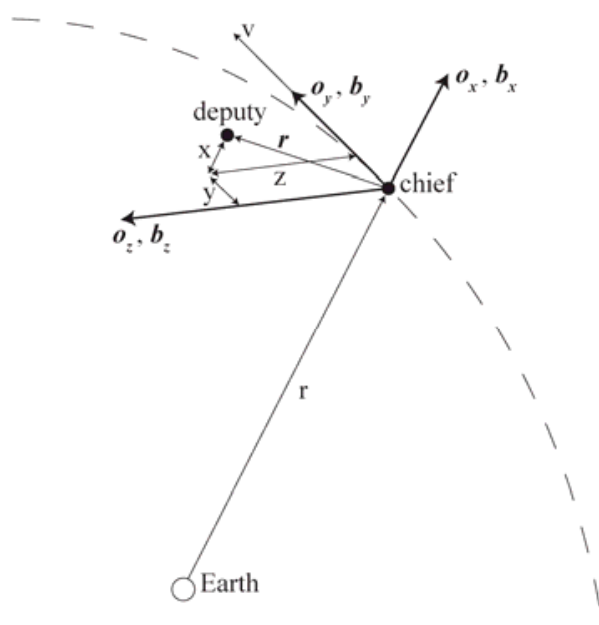
respect to the chief can be expressed through the state

Figure 1: Hill frame and body frame. vector $\boldsymbol{x}$ and its associated set of rectilinear coordinates

$$
\boldsymbol{x}=\left(\begin{array}{lll}
\boldsymbol{r} & \boldsymbol{v}
\end{array}\right)^{T}=\left(\begin{array}{llllll}
x & y & z & \dot{x} & \dot{y} & \dot{z}
\end{array}\right)^{T}
$$

where the vectors $\boldsymbol{r}$ and $\boldsymbol{v}$ denote the relative positions $(x, y, z)^{T}$ and velocities $(\dot{x}, \dot{y}, \dot{z})^{T}$, respectively. In case of a Keplerian two-body motion, a circular chief orbit, and spacecraft separations much smaller than the chief's semi-major axis, the Clohessy-Wiltshire (CW) equations express the linearized relative spacecraft dynamics in the Hill frame [2]:

$$
\begin{aligned}
& \ddot{x}-2 n \dot{y}-3 n^{2} x=a_{x} \\
& \ddot{y}+2 n \dot{x}=a_{y} \\
& \ddot{z}+n^{2} z=a_{z}
\end{aligned}
$$

with $\ddot{x}, \ddot{y}, \ddot{z}$ denoting relative accelerations, $a_{\mathrm{i}}$ perturbing accelerations in $x$-, $y$-, or $z$-direction and $n$ the orbital mean motion of the chief, which is defined as $n=\left(\mu / a^{3}\right)^{1 / 2}$ with $\mu$ the Earth's gravitational constant and $a$ the semi-major axis of the chief's orbit. This system is linear time-invariant, allowing the homogeneous solution to be easily found. The well-known 
solution, in terms of rectilinear coordinates, is provided by [2] as

$$
\boldsymbol{x}(t)=\boldsymbol{\Phi}(t, 0) \boldsymbol{x}(0)=\left(\begin{array}{cccccc}
4-3 c_{n t} & 0 & 0 & s_{n t} / n & \frac{2\left(1-c_{n t}\right)}{n} & 0 \\
6\left(s_{n t}-n t\right) & 1 & 0 & \frac{2\left(c_{n t}-1\right)}{n} & \frac{4 s_{n t}}{n}-3 t & 0 \\
0 & 0 & c_{n t} & 0 & 0 & \frac{s_{n t}}{n} \\
3 n s_{n t} & 0 & 0 & c_{n t} & 2 s_{n t} & 0 \\
6 n\left(c_{n t}-1\right) & 0 & 0 & -2 s_{n t} & 4 c_{n t}-3 & 0 \\
0 & 0 & -n s_{n t} & 0 & 0 & c_{n t}
\end{array}\right)\left(\begin{array}{c}
x(0) \\
y(0) \\
z(0) \\
\dot{x}(0) \\
\dot{y}(0) \\
\dot{z}(0)
\end{array}\right)
$$

with the state transition matrix $\boldsymbol{\Phi}(t, 0)$ mapping the state at time $t_{0}=0$ to the state at time $t, c_{n t}$ equal to $\cos (n t)$ and $s_{n t}$ equal to $\sin (n t)$.

\section{ESTIMATOR}

The observed range $r_{i}$ between the transmitting antenna and receiver antenna $R x_{i}$ at the time of signal reception $t$ is modeled as the Euclidian norm of the relative position vector. The measured pseudorange $\rho$ at time $t$ is modeled as

$$
\begin{aligned}
\rho_{i}(t, \tau) & =r_{i}(t, \tau)+\varepsilon(t)=\left\|\mathbf{r}_{i}(t, \tau)\right\|+\varepsilon(t) \\
& =\sqrt{\left(x(t-\tau)-x_{R x_{i}}(t)\right)^{2}+\left(y(t-\tau)-y_{R x_{i}}(t)\right)^{2}+\left(z(t-\tau)-z_{R x_{i}}(t)\right)^{2}}+\varepsilon(t)
\end{aligned}
$$

with $\tau$ the signal travel time between the transmitting and the receiving antennas and the ranging error $\varepsilon(t)$ modeled as white Gaussian noise with zero mean and standard deviation $\sigma_{\rho}$. Some simplifications can be made in Eq. (4) to reduce the complexity of the problem. Firstly, as the body frame of the chief is aligned with the Hill frame, cf. section 2, the position of the receiving antennas is constant with time. Secondly, the inter-satellite distances and relative velocities considered in the following are small enough to justify setting $\tau$ to zero seconds without introducing significant errors. The pseudorange $\rho$ at time $t$ can then be modeled as

$$
\rho_{i}(t)=\sqrt{\left(x(t)-x_{R x_{i}}\right)^{2}+\left(y(t)-y_{R x_{i}}\right)^{2}+\left(z(t)-z_{R x_{i}}\right)^{2}}+\varepsilon(t) .
$$

We seek to obtain a relative state estimate at time $t_{0}=0$ using as estimator an iterative batch LSQ algorithm. This particular batch LSQ algorithm makes use of a priori information, denoted with the superscript "apr", and is implemented as

$$
\hat{\boldsymbol{x}}_{0}^{j+1}=\hat{\boldsymbol{x}}_{0}^{j}+\Delta \hat{\boldsymbol{x}}_{0}^{j}=\hat{\boldsymbol{x}}_{0}^{j}+\boldsymbol{P}^{j}\left(\Lambda\left(\hat{\boldsymbol{x}}_{0}^{\mathrm{apr}}-\hat{\boldsymbol{x}}_{0}^{j}\right)+\boldsymbol{H}^{* j T} \boldsymbol{W} \Delta \boldsymbol{z}^{j}\right)
$$

with $j$ denoting the number of iterations performed, $\boldsymbol{P}$ the covariance matrix, $\boldsymbol{W}$ a diagonal weighting matrix with $\sigma_{\rho}^{-2}$ on the main diagonal, $\boldsymbol{H}^{*}$ the matrix with the partial derivatives of the modeled observations with respect to the state vector at $t_{0}, \boldsymbol{\Lambda}$ the information matrix (which is the inverse of the a priori covariance matrix $\boldsymbol{P}^{\text {apr }}$ ), and $\Delta \boldsymbol{z}$ the vector with measurement residuals. The covariance matrix $\boldsymbol{P}$ is defined as

$$
\boldsymbol{P}^{j}=\left(\boldsymbol{\Lambda}+\boldsymbol{H}^{* j T} \boldsymbol{W} \boldsymbol{H}^{* j}\right)^{-1}=\left(\left(\boldsymbol{P}^{\mathrm{apr}}\right)^{-1}+\boldsymbol{H}^{* j T} \boldsymbol{W} \boldsymbol{H}^{* j}\right)^{-1} .
$$


Since the state at epoch, $\boldsymbol{x}_{0}$, is to be estimated, the partial derivatives with respect to the state at epoch are needed. This can be achieved, as shown in Eq. (8), by post-multiplying $\boldsymbol{H}_{m}$, the partial derivative matrix at time $m$, with the state transition matrix $\boldsymbol{\Phi}\left(t_{m}, 0\right)$, forming the desired matrix $\boldsymbol{H}^{*}$. The vector $\Delta \hat{\boldsymbol{x}}$ is the difference between the actual state $\boldsymbol{x}$ and the estimated state $\hat{\boldsymbol{x}}$.

$$
\Delta \boldsymbol{z}^{j}=\left(\begin{array}{c}
\Delta z_{0}^{j} \\
\Delta z_{1}^{j} \\
\vdots \\
\Delta z_{m-1}^{j}
\end{array}\right)=\left(\begin{array}{c}
\boldsymbol{H}_{0}^{j} \\
\boldsymbol{H}_{1}^{j} \\
\vdots \\
\boldsymbol{H}_{m-1}^{j}
\end{array}\right) \Delta \hat{\boldsymbol{x}}^{j}=\left(\begin{array}{c}
\boldsymbol{H}_{0}^{j} \boldsymbol{\Phi}\left(t_{0}, 0\right) \\
\boldsymbol{H}_{1}^{j} \boldsymbol{\Phi}\left(t_{1}, 0\right) \\
\vdots \\
\boldsymbol{H}_{m-1}^{j} \boldsymbol{\Phi}\left(t_{m-1}, 0\right)
\end{array}\right) \Delta \hat{\boldsymbol{x}}_{0}^{j}=\left(\begin{array}{c}
\boldsymbol{H}_{0}^{* j} \\
\boldsymbol{H}_{1}^{* j} \\
\vdots \\
\boldsymbol{H}_{m-1}^{* j}
\end{array}\right) \Delta \hat{\boldsymbol{x}}_{0}^{j}=\boldsymbol{H}^{* j} \Delta \hat{\boldsymbol{x}}_{0}^{j}
$$

\section{FACTORS INFLUENCING THE RELATIVE POSITION ESTIMATION ERROR}

In this work, we assume that information on the relative state of the two satellites is obtained by means of pseudorange measurements obtained using a transmitting antenna on the deputy, which is assumed to be a point mass, and either one or three receiving antennas on the chief. In case of three receiver antennas, it is assumed that the locations of the three antennas are known perfectly in the Hill frame, which implies perfect knowledge of the absolute orbit and attitude of the chief. This ranging method is similar to the method used by the FFRF (Formation Flying Radio Frequency) instrument on the currently ongoing PRISMA mission [3].

In this section, equations are derived demonstrating that the ranging error, the inter-satellite distance, and the antenna baseline affect the error in the position determination of a transmitter antenna with respect to a coordinate frame defined by three receiver antennas. The error in the knowledge of the antenna baseline (i.e., the distance between the antenna phase centers) also contributes to this overall error, but is considered to be small enough to be neglected in this study. Subsection 4.1 will define the coordinate frame and all variables needed while subsection 4.2 will develop the equations.

\subsection{Definition of the Receiver Reference Frame and Variables}

The reference frame used here is referred to as the receiver reference frame and is a right-handed Cartesian frame. It is defined by the positions of the three receiver antennas on the chief, as indicated in Figure 2(a). The baselines between the central receiver antenna $\mathrm{Rx}_{0}$ and the receiver antennas $\mathrm{Rx}_{1}$ and $\mathrm{Rx}_{2}$ are indicated as $d_{1}$ and $d_{2}$ respectively. The $x$-axis connects $\mathrm{Rx}_{0}$ with $\mathrm{Rx}_{1} . \mathrm{Rx}_{2}$ is located in the $x y$-plane with its position defined by baseline $d_{2}$ and angle $\alpha . \mathrm{Rx}_{2}$ has been deliberately placed off the $y$-axis to keep the situation general. The direction of the $z$-axis automatically follows from the directions of the $x$-and $y$-axis. Note that this frame can have any (fixed) orientation and offset with respect to the body frame of the chief. We assume that the transmitter antenna Tx is located at coordinates $x_{\mathrm{Tx}}, y_{\mathrm{Tx}}$, and $z_{\mathrm{Tx}}$ in the receiver reference frame, cf. Figure 2(a). The objective is now to find expressions for the coordinates $x_{\mathrm{Tx}}, y_{\mathrm{Tx}}$, and $z_{\mathrm{Tx}}$ using three ranges: $r_{0}, r_{1}$, and $r_{2}$ where $r_{\mathrm{i}}$ is the distance between $\mathrm{Tx}$ and $\mathrm{Rx}_{i}$ for $i=0,1,2$. 


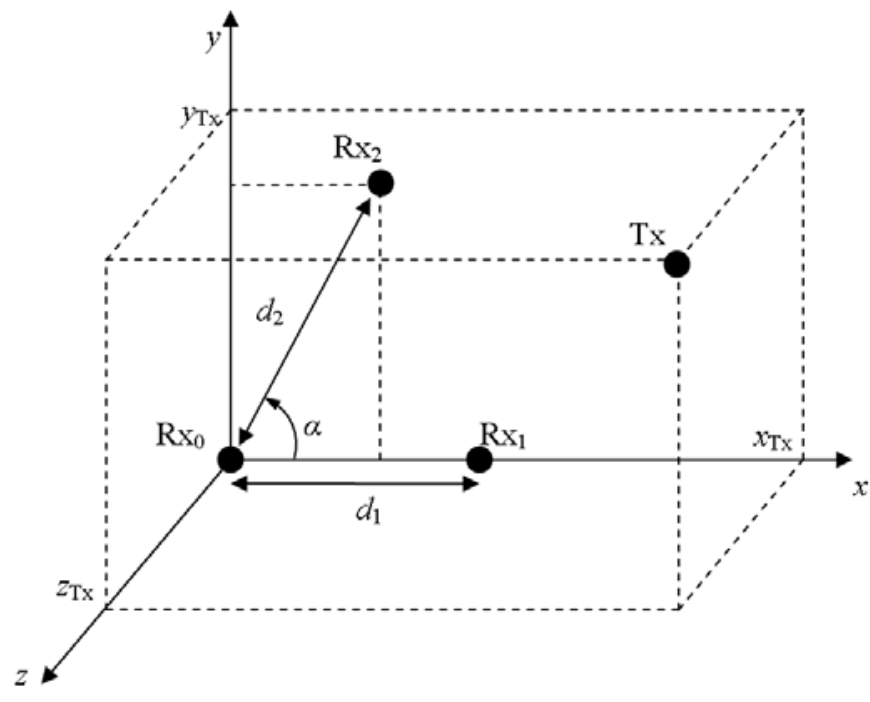

(a)
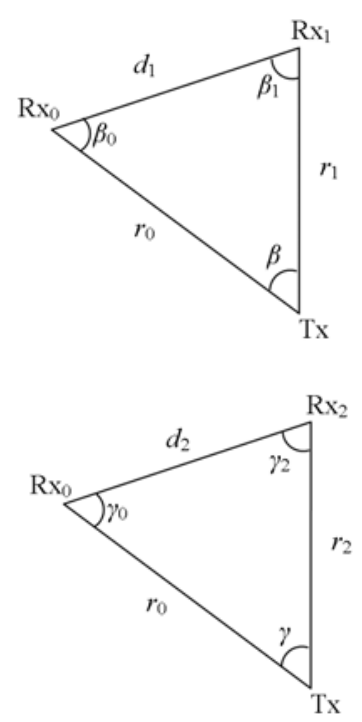

(b)

Figure 2: Location of the various antennas in the receiver reference frame (a) and the definition of the angles in the triangles formed by the Tx and Rx antennas (b).

Since $d_{1}$ and $d_{2}$ are known, two triangles with known side lengths can be formed using the range measurements. The first triangle consists out of vertices $\mathrm{Rx}_{0}, \mathrm{Rx}_{1}$, and $\mathrm{Tx}$ and has side lengths $d_{1}, r_{0}$, and $r_{1}$. The second triangle has vertices $\mathrm{Rx}_{0}, \mathrm{Rx}_{2}$, and $\mathrm{Tx}$ and has side lengths $d_{2}, r_{0}$, and $r_{2}$. Using the cosine rule, all angles of the triangles can be obtained. Using these angles, the coordinates $x_{\mathrm{Tx}}, y_{\mathrm{Tx}}$, and $z_{\mathrm{Tx}}$ can be determined. The angles of the triangles are defined in Figure 2(b).

\subsection{Development of the Equations Expressing the Position of the Transmitting Antenna}

With the side lengths of the triangles known, the various angles in Figure 2(b) can be determined using the cosine rule. Writing all distances in vector form and using the definition of the dot product, the following three equations can be constructed

$$
\begin{gathered}
\cos \beta_{0}=\frac{r_{0}^{2}-r_{1}^{2}+d_{1}^{2}}{2 r_{0} d_{1}}=\frac{\boldsymbol{r}_{0} \bullet \boldsymbol{d}_{1}}{\left|\boldsymbol{r}_{\boldsymbol{0}}\right|\left|\boldsymbol{d}_{1}\right|} \Rightarrow \frac{r_{0}^{2}-r_{1}^{2}+d_{1}^{2}}{2}=\left(\begin{array}{c}
x_{\mathrm{Tx}} \\
y_{\mathrm{Tx}} \\
z_{\mathrm{Tx}}
\end{array}\right) \cdot\left(\begin{array}{c}
d_{1} \\
0 \\
0
\end{array}\right) \\
\cos \gamma_{0}=\frac{r_{0}^{2}-r_{2}^{2}+d_{2}^{2}}{2 r_{0} d_{2}}=\frac{\boldsymbol{r}_{\boldsymbol{0}} \bullet \boldsymbol{d}_{2}}{\left|\boldsymbol{r}_{\boldsymbol{0}}\right|\left|\boldsymbol{d}_{\mathbf{2}}\right|} \Rightarrow \frac{r_{0}^{2}-r_{2}^{2}+d_{2}^{2}}{2}=\left(\begin{array}{c}
x_{\mathrm{Tx}} \\
y_{\mathrm{Tx}} \\
z_{\mathrm{Tx}}
\end{array}\right) \cdot\left(\begin{array}{c}
d_{2} \cos \alpha \\
d_{2} \sin \alpha \\
0
\end{array}\right) \\
\cos \beta=\frac{r_{0}^{2}+r_{1}^{2}-d_{1}^{2}}{2 r_{0} r_{1}}=\frac{\boldsymbol{r}_{0} \bullet \boldsymbol{r}_{1}}{\left|\boldsymbol{r}_{\mathbf{0}}\right|\left|\boldsymbol{r}_{\boldsymbol{1}}\right|} \Rightarrow \frac{r_{0}^{2}+r_{1}^{2}-d_{1}^{2}}{2}=\left(\begin{array}{c}
x_{\mathrm{Tx}} \\
y_{\mathrm{Tx}} \\
z_{\mathrm{Tx}}
\end{array}\right) \cdot\left(\begin{array}{c}
x_{T x}-d_{1} \\
y_{T x} \\
z_{T x}
\end{array}\right) .
\end{gathered}
$$

The expression for $x_{\mathrm{Tx}}$ is obtained from Eq. (9a), leading to Eq. (10a). The expression for $y_{\mathrm{Tx}}$ follows from Eq. (9b) after inserting Eq. (10a), resulting in Eq. (10b). Finally, $z_{\mathrm{Tx}}$ follows from Eq. (9c) after inserting Eq. (10b), resulting in Eq. (10c): 


$$
\begin{gathered}
x_{\mathrm{Tx}}=\frac{r_{0}^{2}-r_{1}^{2}+d_{1}^{2}}{2 d_{1}} \\
y_{\mathrm{Tx}}=\frac{r_{0}^{2}\left(d_{1}-d_{2} \cos \alpha\right)+r_{1}^{2} d_{2} \cos \alpha-r_{2}^{2} d_{1}+d_{1} d_{2}\left(d_{2}-d_{1} \cos \alpha\right)}{2 d_{1} d_{2} \sin \alpha} \\
z_{\mathrm{Tx}}=\frac{\left(\begin{array}{l}
d_{2}^{2} \sin ^{2} \alpha\left[r_{0}^{2}\left(2 r_{1}^{2}-r_{0}^{2}+2 d_{1}^{2}\right)+r_{1}^{2}\left(2 d_{1}^{2}-r_{1}^{2}\right)-d_{1}^{4}\right]+ \\
-\left[r_{0}^{2}\left(d_{1}-d_{2} \cos \alpha\right)+r_{1}^{2} d_{2} \cos \alpha-r_{2}^{2} d_{1}+d_{1} d_{2}\left(d_{2}-d_{1} \cos \alpha\right)\right]^{2}
\end{array}\right)^{1 / 2}}{2 d_{1} d_{2} \sin \alpha} .
\end{gathered}
$$

From Eqs. (10b-c), it is immediately obvious that for $\alpha=0$ no result can be obtained for $y_{\mathrm{Tx}}$ and $z_{\mathrm{Tx}}$ since this condition leads to all receiver antennas being aligned. In addition, the sign of $z_{\mathrm{Tx}}$ is undefined. Using Eqs. (10a-c), the influence of the ranging error, the inter-satellite range, and the antenna baseline on the error in the determination of $x_{\mathrm{Tx}}, y_{\mathrm{Tx}}$, and $z_{\mathrm{Tx}}$ can be determined. First however, it is given without derivation that $\alpha=90^{\circ}$ is the optimum angle (i.e., leading to the smallest position estimation error) between baselines $d_{1}$ and $d_{2}$. This is intuitively obvious as this angle leads to an antenna geometry which is furthest from the situation where all receiver antennas are aligned. Replacing the range $r_{i}$ in Eqs. (10a-c) with the pseudorange $\rho_{i}\left(\rho_{i}=r_{i}+\varepsilon_{i}\right)$, setting $\alpha=90^{\circ}$, and separating the error terms from the error-free terms leads to the estimates $\hat{x}_{\mathrm{Tx}}, \hat{y}_{\mathrm{Tx}}$, and $\hat{z}_{\mathrm{Tx}}$, which are provided for error terms up to first order in Eqs. (11a-c). Note that the ranging error is treated as a bias and not as a random variable, resulting in equations that are valid for a single measurement.

$$
\begin{gathered}
\hat{x}_{\mathrm{Tx}}=x_{\mathrm{Tx}}+\frac{r_{0} \varepsilon_{0}-r_{1} \varepsilon_{1}}{d_{1}}+O\left(\varepsilon^{2}\right) \\
\hat{y}_{\mathrm{Tx}}=\left.y_{\mathrm{Tx}}\right|_{\alpha=90^{\circ}}+\frac{r_{0} \varepsilon_{0}-r_{2} \varepsilon_{2}}{d_{2}}+O\left(\varepsilon^{2}\right) \\
\hat{z}_{\mathrm{Tx}}^{2}=\left.z_{\mathrm{Tx}}^{2}\right|_{\alpha=90^{\circ}}+\frac{\left[\begin{array}{l}
-4 r_{0}^{3} \varepsilon_{0}\left(d_{1}^{2}+d_{2}^{2}\right)-4 r_{1}^{3} d_{2}^{2} \varepsilon_{1}-4 r_{2}^{3} d_{1}^{2} \varepsilon_{2}+ \\
+2 r_{0}^{2}\left(d_{1}^{2} \varepsilon_{2}^{2}\left(2 r_{2}+1\right)+d_{2}^{2} \varepsilon_{1}^{2}\left(2 r_{1}+1\right)-3 \varepsilon_{0}^{2}\left(d_{1}^{2}+d_{2}^{2}\right)\right)+ \\
+2 r_{1}^{2} d_{2}^{2}\left(2 r_{0} \varepsilon_{0}+\varepsilon_{0}^{2}-3 \varepsilon_{1}^{2}\right)+2 r_{2}^{2} d_{1}^{2}\left(2 r_{0} \varepsilon_{0}+\varepsilon_{0}^{2}-3 \varepsilon_{2}^{2}\right)+ \\
+4 r_{0}\left(2 r_{1} d_{2}^{2} \varepsilon_{0} \varepsilon_{1}+2 r_{2} d_{1}^{2} \varepsilon_{0} \varepsilon_{2}\right)+4 d_{1}^{2} d_{2}^{2}\left(r_{1} \varepsilon_{1}+r_{2} \varepsilon_{2}\right)+2 d_{1}^{2} d_{2}^{2}\left(\varepsilon_{1}^{2}+\varepsilon_{2}^{2}\right)
\end{array}\right]+O\left(d_{1}^{2} d_{2}^{2}\right)}{(11)}+
\end{gathered}
$$

The expression for $\hat{z}_{\mathrm{Tx}}$ is very elaborate, but reduces to $\hat{z}_{\mathrm{Tx}}^{2}=r^{2}-d^{2} / 2+\varepsilon(2 r+\varepsilon)$ when assuming $r_{0}=r_{1}=r_{2}=r, d_{1}=d_{2}=\mathrm{d}$, and $\varepsilon_{0}=\varepsilon_{1}=\varepsilon_{2}=\varepsilon$, which is usually approximately true. From Eqs. (11a-c), it is concluded that the error in the determination of the position of the transmitter antenna in the receiver reference frame can be reduced by:

1. increasing the antenna baseline,

2. reducing the inter-satellite range,

3. reducing the ranging error,

4. reducing the difference in the various ranging errors (e.g., ideally, $\varepsilon_{0}=\varepsilon_{1}=\varepsilon_{2}$ ). 
The first three of these are fairly obvious and well known. The fourth one is not directly obvious, but is easily understood. For instance, for the case where Tx is exactly halfway between $\mathrm{Rx}_{0}$ and $\mathrm{Rx}_{1}, r_{0}=r_{1}$ and the triangle formed between $\mathrm{Rx}_{0}, \mathrm{Rx}_{1}$, and $\mathrm{Tx}$ is an isosceles triangle. Then as long as the measured pseudoranges $\rho_{0}$ and $\rho_{1}$ yield the same value, i.e. $\rho_{0}=\rho_{1}$, the triangle that can be formed based on the measurements is also an isosceles triangle, yielding always a correct solution for $x_{\mathrm{Tx}}$. In practice however, it is impossible to obtain the same measurement error for all antennas and can therefore not be designed for.

\section{SIMULATION SCENARIO, RESULTS AND DISCUSSION}

The current section describes the results and setup of simulations performed to investigate the difference in the navigation solution between a single receiver antenna on the chief and three receiver antennas on the chief. The simulation setup is detailed in subsection 5.1, issues with filter divergence are addressed in subsection 5.2 and the final results are provided in subsection 5.3.

\subsection{Simulation Setup}

Since many different formation geometries exist, it is of interest to investigate whether there are differences in the results for different formation geometries. These can be caused by changes in the observability of certain states when the formation geometry is changed. Therefore, we consider several well-known relative orbits which all exhibit bounded 1:1 commensurate motion (i.e., the orbital periods of the chief and deputy match). The baseline relative orbits we investigate are the 2:1 ellipse and the pendulum formation. A 2:1 relative elliptical motion is achieved when the deputy has a slightly different eccentricity than the chief, resulting in a maximum along-track separation in the Hill frame which is twice the maximum radial separation, hence the term 2:1 ellipse. Two varieties of the 2:1 ellipse are studied: the safe ellipse and the projected circular orbit (PCO). When the satellite orbits are coplanar, the relative ellipse is located in the $x y$-plane in the Hill frame. The safe ellipse is created by rotating the coplanar ellipse around the $x$-axis, resulting in a linear motion in the $y z$-plane. This is considered to be a passively safe relative orbit since the cross-track separation and the radial separation never vanish at the same time, hence the term safe ellipse [4]. The PCO is created by rotating the coplanar ellipse around the $y$-axis and sizing the out-of-plane motion such that a circular motion is created in the $y z$-plane. The pendulum formation is basically an along-track formation with added cross-track motion. As such, the along-track formation can be regarded as a limit case for the pendulum formation. Previous work [5] has shown that the out-of-plane states, $z$ and $\dot{z}$, are poorly observable in case of estimation using inter-satellite range measurements. Therefore, the cross-track motion of the formations under study is varied to evaluate its effect on the navigation solution (except for the PCO since any change in the maximum value of $z$ implies a similar change in $x$ and $y$ as well, which is not desired since it greatly affects the inter-satellite range). The formations under consideration are summarized in Table 1. The orbit of the chief is circular with a semi-major axis of $7028 \mathrm{~km}$. 


\begin{tabular}{ccccccccc}
\hline \multicolumn{1}{c}{ Formation } & \multicolumn{7}{c}{ Relative state elements at $\boldsymbol{t}_{\mathbf{0}}$} \\
\hline \hline & $x_{0}[\mathrm{~m}]$ & $y_{0}[\mathrm{~m}]$ & $z_{0}[\mathrm{~m}]$ & $\dot{x}_{0}$ & {$[\mathrm{~m} / \mathrm{s}]$} & $\dot{y}_{0}[\mathrm{~m} / \mathrm{s}]$ & $\dot{z}_{0}[\mathrm{~m} / \mathrm{s}]$ \\
\hline Safe ellipse 1 & 100 & 0 & 0 & 0 & $-2 n x_{0}$ & $-n x_{0}$ \\
PCO & $\sqrt{2000}$ & 0 & $\sqrt{8000}$ & 0 & $-2 n x_{0}$ & 0 \\
Pendulum 1 & 0 & -100 & 0 & 0 & 0 & $-n y_{0}$ \\
Safe ellipse 2 & 100 & 0 & 0 & 0 & $-2 n x_{0}$ & $-0.01 n x_{0}$ \\
Pendulum 2 & 0 & -100 & 0 & 0 & 0 & $-0.01 n y_{0}$ \\
\hline
\end{tabular}

Table 1: Summary of the formations studied (for the smallest inter-satellite range)

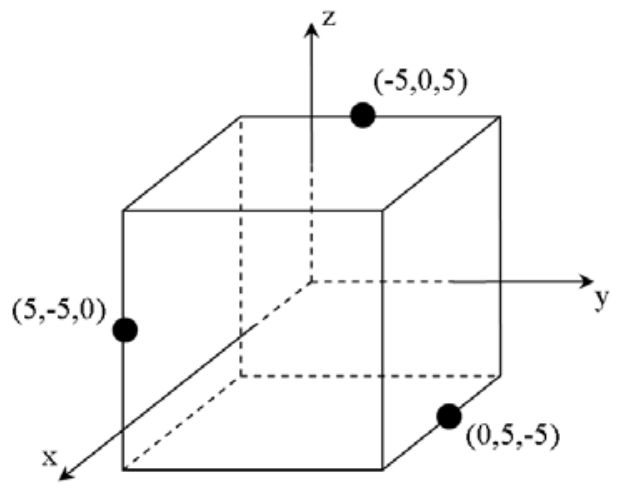

Figure 3: Antenna locations on the chief in the Hill frame.

To investigate the influence of receiver antenna baseline, ranging accuracy, and inter-satellite distance on the navigation solution, these variables are varied in magnitude. For all formation geometries investigated, each of these variables is given three different values, which differ one order of magnitude with respect to each other (i.e., 1-10-100). This leads to a total of 36 cases considered for each relative orbit. For the multiple receiver antenna case, the location of the antennas on the chief is deliberately chosen such that a homogeneous distribution over the satellite body, assumed to be cubic, is achieved, cf. Figure 3. The side length of the chief can be either $10 \mathrm{~cm}, 10 \mathrm{dm}$, or $10 \mathrm{~m}$, leading to antenna baselines of $(150)^{1 / 2} \mathrm{~cm},(150)^{1 / 2} \mathrm{dm}$, or $(150)^{1 / 2} \mathrm{~m}$, respectively. In case of a single receiver antenna, the chief is assumed to be a point mass. The ranging accuracy is varied between $1 \mathrm{~cm}, 1 \mathrm{dm}$, and $1 \mathrm{~m}$. The inter-satellite distance at epoch, $r_{\mathrm{e}}$, is varied between $100 \mathrm{~m}, 1 \mathrm{~km}$, and $10 \mathrm{~km}$. The error in the a priori state estimate is set equal to $0.001 r_{\mathrm{e}}$ in relative position and $0.001 \mathrm{nr}$ e in relative velocity for all formations studied. The a priori estimation error has been deliberately kept small to prevent the estimator from converging to an ambiguous solution for the relative state estimate for the elliptical formation geometries in case of long measurement arcs. This occurs for both sensor sets at the same measurement arc length, irrespective of the measurement accuracy, antenna baseline or inter-satellite distance. The a priori standard deviations are set to $0.1 r_{\mathrm{e}}$ in relative position and $0.1 n r_{\mathrm{e}}$ in relative velocity. Each receiver antenna performs 100 range measurements per orbit for a maximum of five orbits and the estimator performs state estimations for measurement arc lengths of 0.1 to 5 orbits with steps of 0.1 orbits. 


\subsection{Filter Divergence due to Nonlinearities}

The reference trajectory, from which the inter-satellite range measurements are generated, is obtained by integration of the $\mathrm{CW}$ equations. Although it is recognized that this approach does not reflect the true relative motion of the satellites, it is necessary to avoid filter divergence (i.e., state estimation errors much larger than the standard deviation in the state estimate) caused by nonlinearities. The reason for this is explained in the following. In the simulations performed, first the 'true' relative state at epoch is defined and is subsequently propagated in time using appropriate dynamics. Range measurements are then constructed by calculating the distances between the transmitting and receiving antennas and adding a random measurement error. In the estimator, the same procedure is followed, but then using an estimate for the relative state and without adding noise to the predicted measurements. The estimator then uses the difference between the 'true' measurements and the predicted measurements to improve the state estimate using the presented batch LSQ algorithm until a certain convergence criterion is satisfied. This process is depicted schematically in Figure 4.

It is commonly preferred to propagate the estimated relative orbit using the $\mathrm{CW}$ equations since this does not require any information on the absolute satellite orbits except for the semi-major axis of the chief. Propagation of the reference trajectory can now either be done using the $\mathrm{CW}$ equations or by propagating the absolute orbits of both satellites and determining the relative motion in the Hill frame using a standard transformation. Initially, the latter option, assuming purely Keplerian dynamics, was selected since this provides a result closer to reality. However, this led to filter divergence for large inter-satellite distances and high measurement accuracy, cf. Figure 5. In Figure 5, the evolution of the estimation error and the corresponding standard deviation for $x_{0}$ is depicted for a safe elliptical formation with dimensions of $1 \times 2 \times 1 \mathrm{~km}$ when using a single receiver antenna. The ranging accuracy is $1 \mathrm{~cm}$, the a priori estimation error is zero for all states and the a priori standard deviations are $1 \mathrm{~m}$ for all relative positions and $1 \mathrm{n} \mathrm{m} / \mathrm{s}$ for all relative velocities. In Figure 5(a), depicting the results when the reference trajectory is generated by propagating the absolute Keplerian orbits of the satellites, a clear spike in the estimation error is visible for a data batch of 0.9 orbits. However, there is no similar spike in the standard deviation. Figure 5(b) depicts the results when using the $\mathrm{CW}$ equations to propagate the reference trajectory. There, the result is what it should be with the estimation error staying well within the $3 \sigma$ bounds.

The cause of this behavior lies in the fact that the $\mathrm{CW}$ equations are linearized while the Keplerian dynamics are not. This leads to small differences in the range between the satellites for both methods. For inter-satellite distances in the order of $\mathrm{km}$, the neglected nonlinearities are on the order of $\mathrm{cm}$, leading to discrepancies between the predicted and the 'true' measurements in this case. Because the measurements are very accurate, the measurement weight is fairly large, causing the state estimate to be pulled towards a solution that closely matches the measurements, but which is erroneous since the $\mathrm{CW}$ dynamics differ from the Keplerian dynamics. For larger inter-satellite distances and higher measurement accuracy, this effect gets more and more pronounced, leading to ever worsening results. Note that this effect also appears in case of multiple receiver antennas, irrespective of the antenna baseline. A more accurate representation of the relative motion instead of the $\mathrm{CW}$ equations in the estimator would relieve this problem, but as the goal of this study is to compare different receiver antenna configurations and not to achieve the best relative position estimation result, it was decided to propagate both the reference trajectory and the estimated trajectory using the $\mathrm{CW}$ equations. 


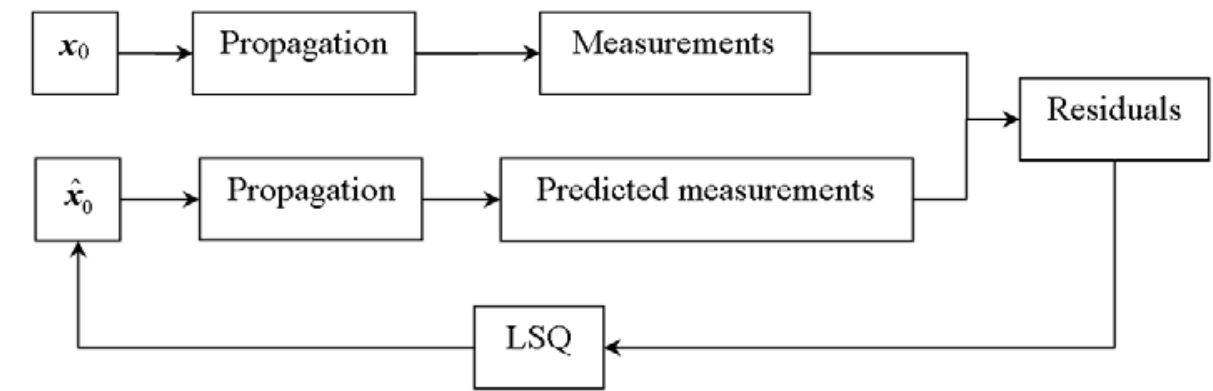

Figure 4: Schematic representation of the relative state estimation process.

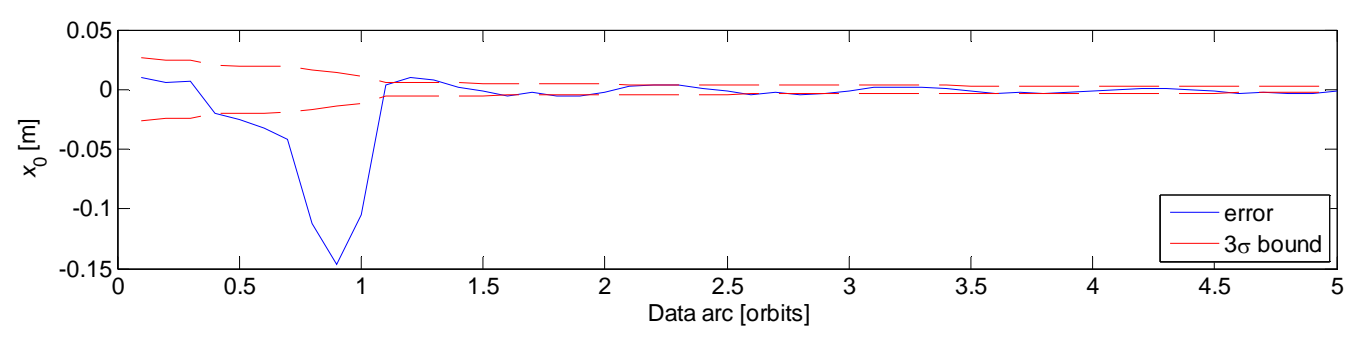

(a)

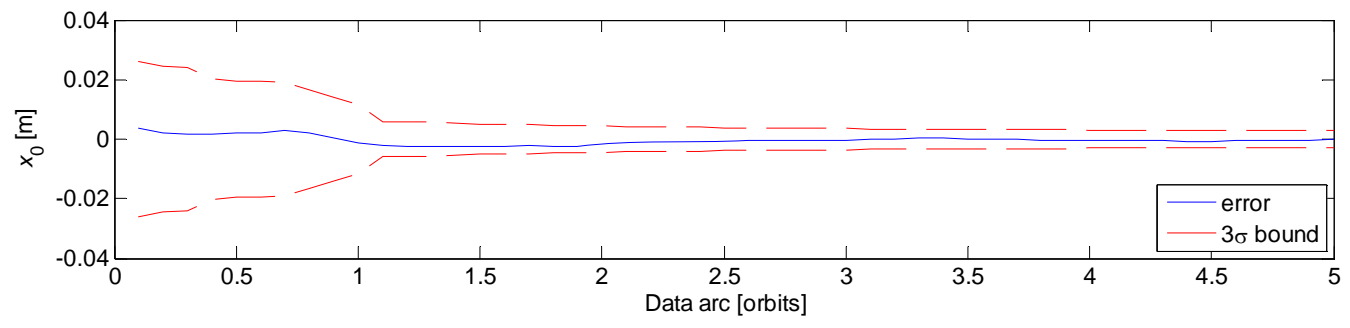

(b)

Figure 5: State estimation results for a $1 \times 2 \times 1 \mathrm{~km}$ safe ellipse when propagating the relative orbit using Keplerian dynamics (a) and when propagating the relative orbit using the $\mathrm{CW}$ equations (b). The measurement accuracy is $1 \mathrm{~cm}$.

\section{$\underline{5.3 \text { Simulation Results }}$}

Due to the sheer number of variables considered, the simulation results are not presented for all individual states. Instead, only the three-dimensional root sum squared (3D RSS) standard deviation, $\sigma_{\mathrm{RSS}}$, in the relative position estimation error is provided. This figure of merit is considered to provide sufficient information on the convergence of the total state estimate. Special results for particular states will be highlighted when applicable. Table 2 provides the approximate estimation results for the first safe ellipse, the PCO, and the first pendulum formation for a measurement batch of 5 orbits. These are provided as a function of the a priori standard deviation in the position estimate, ranging accuracy, antenna baseline, and initial inter-satellite distance.

Analyzing the first two rows of Table 2 it is observed that for elliptical formation geometries the single receiver antenna case results in failure of the estimator to converge to small standard deviations. This is caused by $y, z$, and $\dot{x}$ being poorly observable, causing the standard deviation in their estimation to hardly improve with respect to their a priori standard deviations. For the sensor set with three receiver antennas the result is interesting. The result depends on the ratio of the antenna baseline and the ranging accuracy: As soon as the ranging accuracy is of the same order of magnitude as the antenna baseline, the three antennas effectively behave as a single antenna, causing $y, z$, and $\dot{x}$ again to be poorly observable 
and leading to a navigation solution comparable to that of the single receiver antenna. For the sensor set with three receiver antennas, a larger antenna baseline always leads to a better result and a ten times larger inter-satellite distance leads to a ten times poorer navigation solution.

For the first pendulum formation all states are well observable for all sensor sets, leading to a proper convergence of the estimate, as can be observed from the third row of Table 2 . Contrary to the elliptical formation geometry, the inter-satellite distance and the receiver antenna baseline have no effect on the accuracy of the navigation solution. The ranging accuracy however does have an influence: The accuracy in the navigation solution is always roughly one order of magnitude better than the ranging accuracy. The difference between three receiver antennas and one receiver antenna is caused by the total number of measurements made by a sensor set, which is three times more for the sensor set with three receiver antennas, which causes the factor (3) $)^{1 / 2}$ improvement over the single receiver antenna case. Thus, for the pendulum formation, three receiver antennas do not improve the geometry of the problem.

\begin{tabular}{|c|c|c|}
\hline Formation & Single receiver antenna & Three receiver antennas \\
\hline Safe ellipse 1 & $\sigma_{\mathrm{RSS}}=\sigma^{\mathrm{apr}}$ & $\left\{\begin{array}{l}\sigma_{\mathrm{RSS}}=\sigma^{\mathrm{apr}} \quad \text { if } d<\sigma_{\rho} \\
\sigma_{\mathrm{RSS}}=\frac{2}{3} \sigma^{\mathrm{apr}} \text { if } d \approx \sigma_{\rho} \\
\sigma_{\mathrm{RSS}}=\frac{r_{\mathrm{e}} \sigma_{\rho}}{10 d} \text { if } d>\sigma_{\rho}\end{array}\right.$ \\
\hline $\mathrm{PCO}$ & $\sigma_{\mathrm{RSS}}=\sigma^{\mathrm{apr}}$ & $\left\{\begin{array}{l}\sigma_{\mathrm{RSS}}=\sigma^{\mathrm{apr}} \quad \text { if } d<\sigma_{\rho} \\
\sigma_{\mathrm{RSS}}=\frac{2}{3} \sigma^{\mathrm{apr}} \text { if } d \approx \sigma_{\rho} \\
\sigma_{\mathrm{RSS}}=\frac{r_{\mathrm{e}} \sigma_{\rho}}{10 d} \text { if } d>\sigma_{\rho}\end{array}\right.$ \\
\hline Pendulum 1 & $\sigma_{\mathrm{RSS}}=\frac{\sigma_{\rho}}{5}$ & $\sigma_{\mathrm{RSS}}=\frac{\sigma_{\rho}}{5 \sqrt{3}}$ \\
\hline Safe ellipse 2 & $\sigma_{\mathrm{RSS}} \approx \sigma^{\mathrm{apr}}$ & $\left\{\begin{array}{l}\sigma_{\mathrm{RSS}} \approx \sigma^{\mathrm{apr}} \quad \text { if } d<\sigma_{\rho} \\
\sigma_{\mathrm{RSS}} \approx \frac{2}{3} \sigma^{\mathrm{apr}} \text { if } d \approx \sigma_{\rho} \\
\sigma_{\mathrm{RSS}} \approx \frac{r_{0} \sigma_{\rho}}{10 d} \text { if } d>\sigma_{\rho}\end{array}\right.$ \\
\hline Pendulum 2 & $\sigma_{\mathrm{RSS}}=\frac{50 \sigma_{\rho}}{4}$ & $\sigma_{\mathrm{RSS}}=\frac{50 \sigma_{\rho}}{4 \sqrt{3}}$ \\
\hline
\end{tabular}

Table 2: Approximate 3D RSS Standard deviation in the position estimate for a batch size of five orbits. 

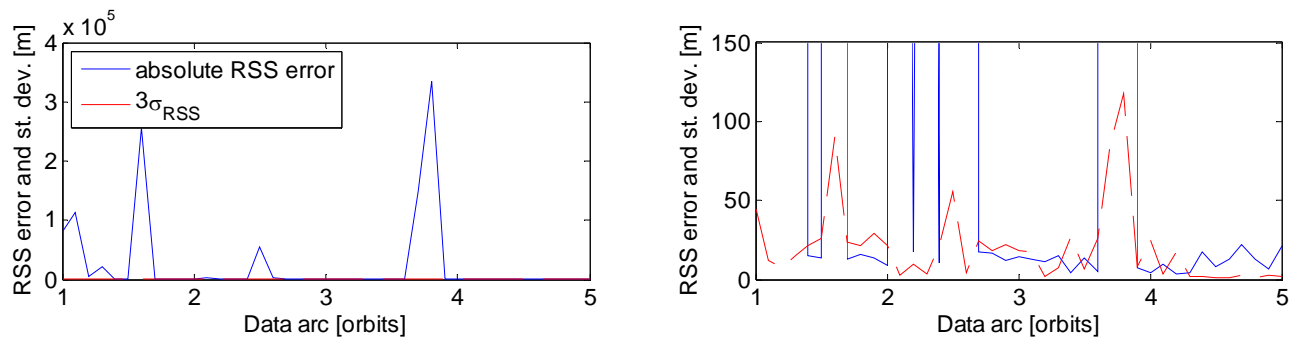

Figure 6: Selected navigation solution for the second safe ellipse. The figure on the right is a zoomed-in version of the figure on the left.

The result for the safe ellipse and the PCO is very similar, but very different from the result for the pendulum. The reason for this is that the pendulum formation only exhibits a cross-track motion, which causes the out-of-plane states $z$ and $\dot{z}$ to be relatively well-observable, even for the single receiver antenna case. From [5], it is known that these states are the least observable states in case of limited cross-track motion and a sensor set containing only a single receiver antenna. Simulations performed for the second safe ellipse and the second pendulum formation have confirmed that this is also true for the sensor set with three receiver antennas. For the second pendulum formation, the accuracy in the solution is sixty times worse when compared to the first pendulum formation, which is caused by the now very limited cross-track motion. For the second safe ellipse however, the estimation result is comparable to that of the first safe ellipse, even though also here there is very limited cross-track motion. The reason for this lies in the fact that there is still plenty of motion in radial and along-track direction, which clearly helps in obtaining an accurate solution However, whereas the result for the first safe ellipse varied smoothly between data batches of different size, the result can now be much more erratic and can even become unstable for large inter-satellite distances and small ranging errors, leading to wrong solutions due to a singular normal equations matrix, cf. Figure 6 . There, the 3D RSS position error and standard deviation are provided for a case with three receiver antennas, $r_{\mathrm{e}}=1000 \mathrm{~m}, \sigma_{\rho}=0.01 \mathrm{~m}$, $d=(150)^{1 / 2} \mathrm{~cm}$ and data batches ranging from 0.1 to 5 orbits. This can only be prevented by reducing the magnitude of the a priori standard deviations for the out-of-plane states. Reducing these with a factor 100 results in a stable, albeit erratic, solution again. Therefore, it is concluded that relative navigation using inter-satellite ranging for formations with coplanar orbits is unreliable if the a priori information on the out-of-plane states is of poor quality.

\section{CONCLUSIONS}

The estimation of the relative state of two formation flying satellites is performed for two different sensor sets. Both sets directly measure the inter-satellite range using radiofrequency ranging signals and one transmitter antenna. However, one set only uses a single receiver antenna while the other set uses three receiver antennas. It is shown analytically that the accuracy in the estimation of the position of a transmitter with respect to a reference frame defined by three receiving antennas is affected by the distance between the transmitter and the receivers, the baseline between the receivers, and the ranging accuracy. In light of these findings, it is of interest to determine whether a single receiver antenna, which is generally less costly and less complex to implement than three receiver antennas, can achieve a navigation performance comparable to the more complete sensor set. Several formation geometries are investigated and for each formation the ranging accuracy, antenna baseline, and inter-satellite distance is varied to study their effects on the navigation solution accuracy. The range measurements are treated together with a dynamic model of the satellites' relative motion to estimate their relative orbit using an iterative batch least-squares algorithm. 
For any elliptical formation geometry with considerable out-of-plane motion, three receiver antennas outperform a single receiver antenna as long as the ranging accuracy is smaller than the receiver antenna baseline. If not, the root sum squared (RSS) standard deviations in the relative position estimate become similar to those for the single receiver antenna case, irrespective of inter-satellite distance. This is caused by the poor observability of the states $y, z$, and $\dot{x}$ for this formation geometry, causing the standard deviation in their estimation to improve little beyond its a priori value.

For a pendulum formation, the inter-satellite distance and the receiver antenna baseline have no effect on the accuracy of the navigation solution. The ranging accuracy however does have an influence: The accuracy in the navigation solution scales linearly with the ranging accuracy. Since the antenna baseline does not affect the accuracy in the navigation solution, three receiver antennas do not provide any geometrical advantage over a single receiver antenna. The only benefit they offer is statistical: three antennas lead to three times more measurements being made, resulting in a factor $(3)^{1 / 2}$ improvement in accuracy.

Decreasing the magnitude of the out-of-plane motion deteriorates the accuracy of the navigation solution for a pendulum formation in a linear fashion. This is caused by the out-of-plane state components being poorly observable and a motion in the cross-track direction only. For elliptical formation geometries, reducing the magnitude of the out-of-plane motion does not deteriorate the accuracy in the navigation solution, even though the out-of-plane state components are also here poorly observable. The reason for this is the large remaining motion in the radial and along-track direction, which is sufficient to lead to an accurate solution. However, in case of very small out-of-plane motion and large a priori standard deviations for those states, the state estimation may become unstable for an elliptical formation geometry due to a near-singular normal equations matrix. This phenomenon can be relieved by decreasing the a priori covariances for the out-of-plane states.

\section{REFERENCES}

1. Hill, G.W., "Researches in the Lunar Theory", American journal of Mathematics, Vol. 1, 1878 , pp. 5-26.

2. Alfriend, K.T, Vadali, S.R., Gurfil, P., How, J.P., Breger, L.S., "Spacecraft Formation Flying - Dynamics, control and navigation", $1^{\text {st }}$ Edition, Butterworth-Heinemann, Kidlington, UK, 2010, ISBN 978-0-75-068533-7.

3. Harr, J., Delpech, M., Grelier, T., Seguela, D., Persson, S.,"The FFIORD experiment CNES' RF metrology validation and formation flying demonstration on PRISMA", $3^{\text {rd }}$ International Symposium on Formation Flying Missions and Technologies, Noordwijk, The Netherlands, April 23-25, 2008.

4. D'Amico, S. and Montenbruck, O., "Proximity Operations of Formation-Flying Spacecraft Using an Eccentricity/Inclination Vector Separation", Journal of Guidance, Control \& Dynamics, Vol. 29, No. 3, 2006, pp. 554-563.

5. Maessen, D and Gill, E.," Relative Orbital Element Estimation and Observability Analysis for Formation Flying Satellites using Inter-Satellite Range Measurements Only", paper AIAA-2010-7885, AIAA Guidance, Navigation, and Control Conference, Toronto, Canada, August 2-5, 2010. 